\title{
Split Suction Catheter Stuck in an Endotracheal Tube: A Case Report
}

\author{
Soo Yeon Kim, Kyu Nam Kim*, Sun Jung Eun, Min Kyu Lee and Mi Sun Kang \\ Department of Anesthesiology and Pain Medicine, Hanyang University Hospital, Republic of Korea
}

\begin{abstract}
Endotracheal tube (ETT) suction is frequently required to remove a mucosal plug or clots to prevent airway obstruction. We encountered a case of a suction catheter that was stuck in an ETT and split by forcible pulling. The patient was intubated with a $6.5 \mathrm{~mm}$ oral-preformed ETT. At the end of surgery, a 12-French suction catheter became lodged in the ETT. The catheter was pulled forcibly, and it split into two parts. Normal saline was injected into the ETT for lubrication and, using forceps, the catheter was pulled in small increments and successfully removed. To prevent this complication, physicians should carefully select the appropriate catheter size, and the catheter should be lubricated. If a suction catheter becomes stuck in an ETT, instillation of normal saline and use of forceps may facilitate removal.
\end{abstract}

\section{Keywords}

Airway management, Catheters, Lubrication, Suction

\section{Introduction}

Suction of the endotracheal tube (ETT) is frequently required for mechanically ventilated patients in the operating room or intensive care unit. It can efficiently remove a mucosal plug or clots, and it maintains airway patency by preventing airway from narrowing or clogging. However, several complications related to ETT suction have been described [18]. We report this unusual case of a suction catheter stuck in an ETT and split by forcibly pulling.

\section{Case Report}

An 89-year-old female, height $158 \mathrm{~cm}$ and weight $60 \mathrm{~kg}$, was scheduled for emergency ophthalmic surgery due to traumatic eyeball rupture. The patient had controlled hypertension and Parkinson's disease with American Society of Anesthesiologists physical status IIE. On preoperative examination, serum potassium level was $2.6 \mathrm{mEq} / \mathrm{L}$, and all other laboratory findings were normal. Her chest radiograph showed an irregular opacity at the peripheral lung field.

Anesthesia was induced by $1 \%$ lidocaine, propofol, and rocuronium and was maintained with desflurane, oxygen, air, and remifentanil. The patient was intubated with a $6.5 \mathrm{~mm}$ internal diameter (ID) oral-preformed ETT (Mallinckrodt RAE oral ${ }^{\circledR}$, Covidien, Ireland). The surgery lasted for approximately one hour. At the end of surgery and before emergence from anesthesia, suction of the ETT was attempted. A 12-Fr suction catheter (PVC and latex, Insung Medical, South Korea) was wiped with an alcohol swab and inserted through the ETT with some resistance. After connecting vacuum tubing to the catheter, suction was applied. Thereafter, despite efforts to remove it, the suction catheter was lodged in the ETT. Because the patient could not be ventilated with the suction catheter stuck within the ETT, the catheter was pulled forcefully and eventually stretched and split into two parts. Immediately, the ETT was connected to the ventilator with the distal part of the suction catheter remaining within the ETT. The patient was well ventilated without any signs of airway obstruction, and peripheral oxygen saturation remained stable. We prepared Jackson extra lightweight cup forceps (Figure 1, Teleflex Medical, Research Triangle Park, USA), and $1 \mathrm{ml}$ of normal saline was injected into the ETT for lubrication. Using the forceps, the catheter was repeatedly pulled in small increments. As the catheter approached the tube inlet, it was grasped by a Kelly forceps. Finally, the catheter was successfully removed. It was found that mucosal secretions had adhered to the distal tip of the catheter (Figure 2). The sum of the lengths of the two split parts of the catheter was longer than the length of a new catheter, which means that the catheter was stretched before it split. The patient's vital

*Corresponding author: Kyu Nam Kim, MD, PhD, Department of Anesthesiology and Pain Medicine, Hanyang University Hospital, 222, Wangsimni-ro, Seongdonggu, Seoul, 04763, Republic of Korea, Tel: 822-2290-8680, Fax: 822-2299-8692

Accepted: August 20, 2019

Published online: August 22, 2019

Citation: Kim SY, Kim KN, Eun SJ, et al. (2019) Split Suction Catheter Stuck in an Endotracheal Tube: A Case Report. J Clin Anesth Pain Manag 3(1):82-84 


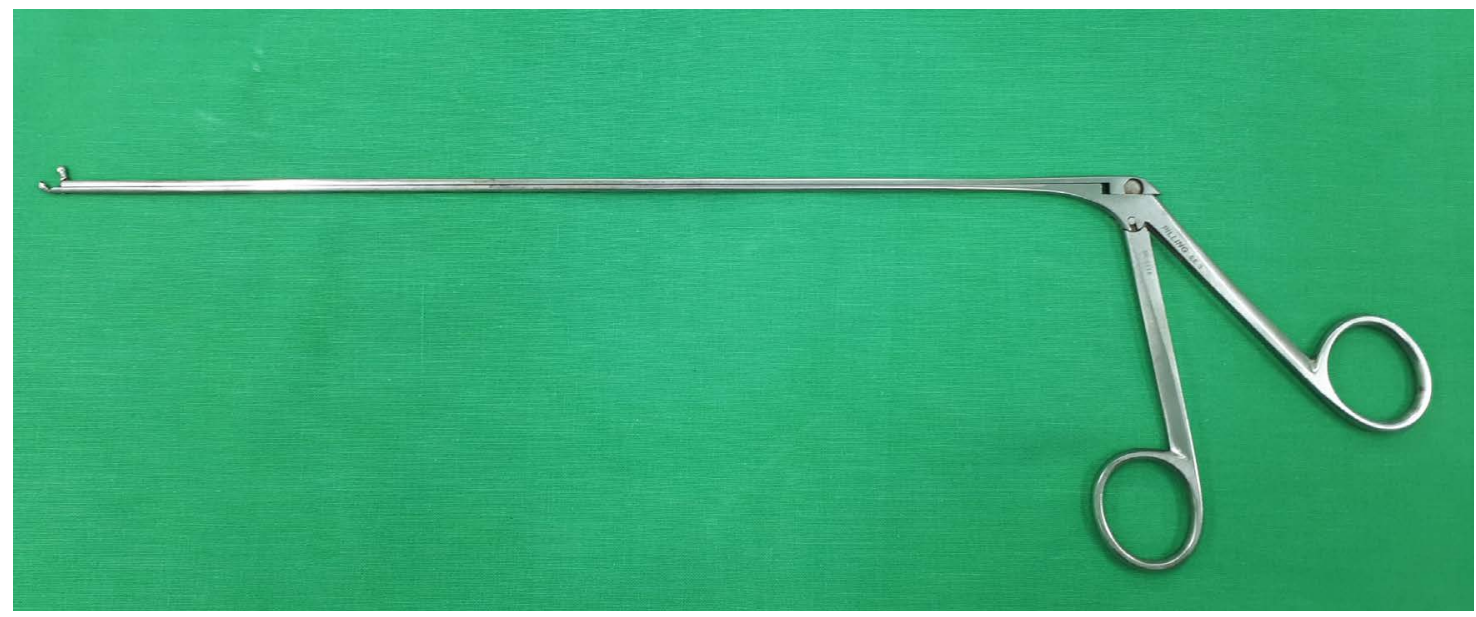

Figure 1: Jackson extra lightweight cup forceps (Teleflex Medical, Research Triangle Park, North Carolina, USA).

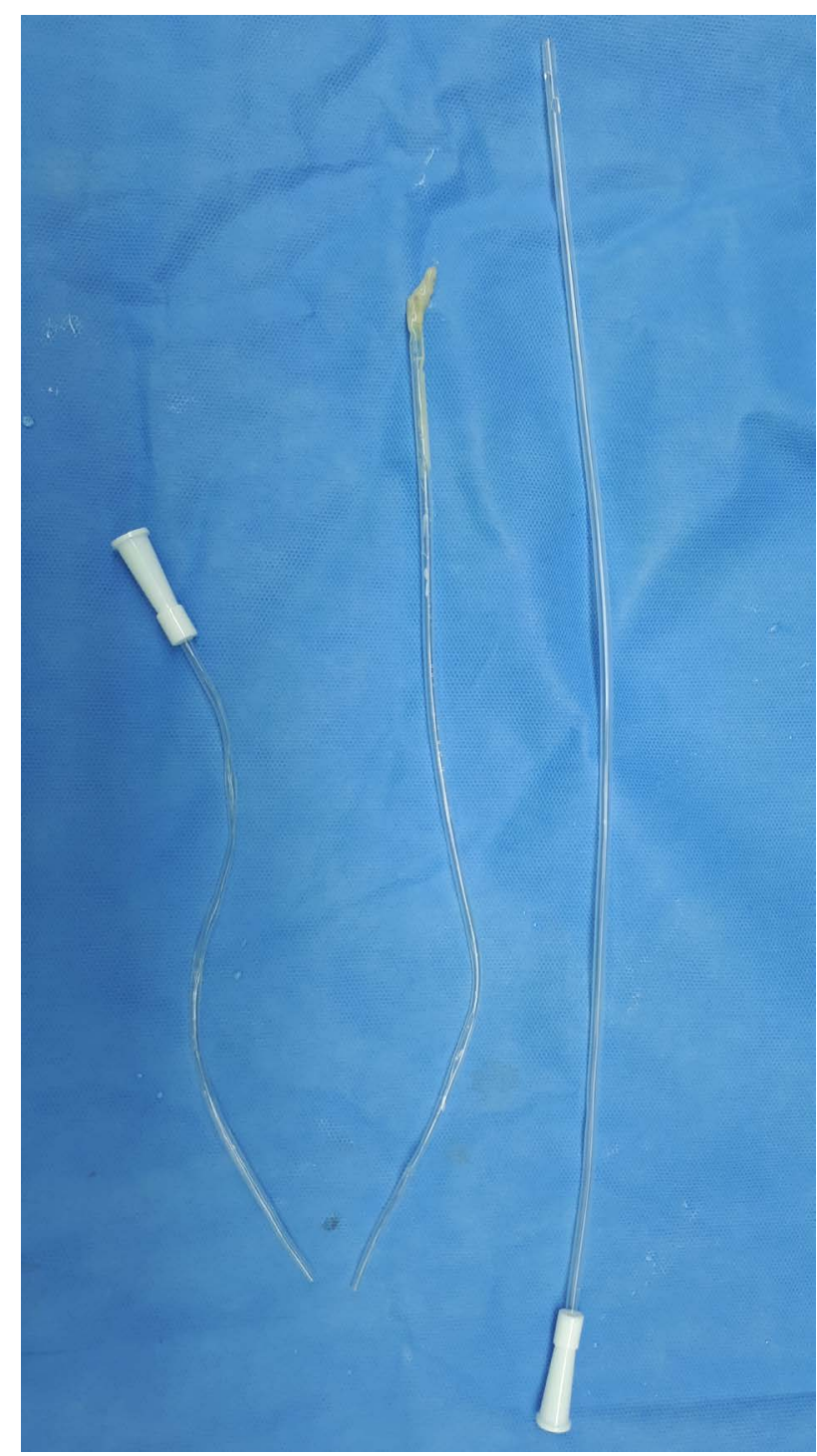

Figure 2: Split parts of the suction catheter (left, middle) and a new one (right). Mucosal secretion adhered to the distal tip of the catheter. signs were stable throughout the event, and the patient was moved to the general ward without any complications.

\section{Discussion}

ETT suctioning is a necessary procedure for mechanically ventilated patients, but it can cause some hazardous complications, such as extreme impaction or kinking of the suction catheter [1-8]. In the present case, a suction catheter became stuck in the ETT and was split by forcible pulling. The situation may be attributable to the following factors. First, an oral preformed ETT was used because of the type of surgery. The preformed ETT has more extreme curvature than the standard ETT, interrupting the passage of the suction catheter. Second, the secretion discovered at the end of the catheter may have caused the catheter to stick to the ETT. In addition, the catheter was wiped with an alcohol cotton swab, and the moisture layer might have caused increased adhesive forces between the catheter and the ETT. Raut, et al. [2] and Singh and Chugh [8] suggested that a thin moisture layer, due to water condensation along the inner wall of ETT, caused close contact between the ETT and the catheter.

Once the suction catheter becomes stuck in the ETT, the situation can be very dangerous because the lodged catheter may interrupt the connection between the ETT and the ventilator circuit. To prevent this situation, the suction catheter should be lubricated. Taylor, et al. [9] reported that use of any lubricants reduced the work needed to retract the stylet from a tracheal tube compared to non-lubrication. On this basis, it is reasonable to assume that lubrication of a suction catheter will facilitate the passage of the suction catheter through the ETT. In addition, an appropriate catheter size should be selected according to the size of the tracheal tube. The American Association for Respiratory Care (AARC) clinical practice guidelines for endotracheal suction of mechanically ventilated patients with airway suggest the use of a suction catheter that occludes less than $50 \%$ of the lumen of the ETT in children and adults and less than $70 \%$ in infants [10]. The 12-Fr suction catheters are $4 \mathrm{~mm}$ in outer diameter, and the suction catheter used in the present case occupied $37.8 \%$ of the $6.5 \mathrm{~mm}$ ID ETT. Although we used an 
appropriately-sized suction catheter, it still became lodged in the ETT. The reason for this is presumed to be that the cross section of the curved part of the preformed ETT was triangular rather than circular. Lastly, if some resistance is detected when the suction catheter enters the ETT, one should not insert the catheter forcibly to avoid the impaction of the suction catheter.

If the catheter lodges in the tracheal tube despite these efforts, it might be helpful to rotate the catheter or to change the curvature of the tracheal tube. It is important to avoid forcible pulling because the catheter can be divided into two parts. The remaining part of the catheter can migrate into the distal airway, necessitating bronchoscopic foreign body removal. Removing the ETT with the catheter in situ, rather than continuing the efforts to pull out the suction catheter, can quickly resolve the problem, but there has been a case of failed re-intubation [1-8]. Therefore, as in our case, instillation of normal saline and application of instruments, such as forceps, might be a good option.

In conclusion, suctioning of the tracheal tube occasionally causes impaction of the suction catheter and a subsequent dangerous situation, especially when a preformed ETT is used. To reduce the risk of such an event, the appropriate catheter size should be selected, and the catheter should be lubricated. The practitioner should not forcibly insert or pull the catheter when resistance is detected. If a suction catheter becomes stuck in the ETT, instillation of normal saline and use of forceps may facilitate removal.

\section{References}

1. Shah PJ, Ray A, Verma S (2017) An unforeseen complication of suctioning an endotracheal tube having murphy's eye. Cent J ISA 1: 81-82.

2. Raut M, Maheshwari A, Joshi (2015) Stuck suction catheter in endotracheal tube. Indian J Crit Care Med 19: 113.

3. Aggarwal R (2014) Suction catheter impaction: An airway emergency. Anesth Essays Res 8: 417.

4. Dubey PK, Sanjeev OP (2013) Impaction of suction catheter during paediatric anaesthesia. Anaesthesia 68: 109-110.

5. Gupta A, Mohta A, Kamal G, et al. (2010) Impaction of suction catheter - Complication of endotracheal suctioning. Indian J Crit Care Med 14: 222.

6. Jagannathan N, Pak TY (2009) An unusual complication of endotracheal tube suctioning. J Anesth 23: 170-171.

7. Takrouri M, Nafakh R, Abbas A (2008) Suction Catheter Impaction In Preformed Nasal Endotracheal Tube (PNETT) During Pediatric Dental Anesthesia - Hazard Notice. Internet J Anesthesiol 22: 1-4.

8. Singh B, Chugh V (2002) An unusual complication of suction through the tracheal tube. Anaesthesia 57: 718.

9. Taylor AM, Hung OR, Kwofie K, et al. (2012) Removal of the stylet from the tracheal tube: Effect of lubrication. Anaesthesia 67: $885-888$.

10. American Association for Respiratory Care (2010) AARC Clinical Practice Guidelines. Endotracheal suctioning of mechanically ventilated patients with artificial airways 2010. Respir Care 55: 758-764.

DOI: $10.36959 / 377 / 332$ 